\title{
Optimal Packet Loss Rate Based Vertical Handoff Algorithm for Wireless Heterogeneous Networks
}

\author{
S. Shibu \\ Assistant Professor, Department of ECE, Panimalar Engineering College, Chennai, India
}

\begin{abstract}
The Vertical handover algorithm allows the mobile wireless device to roam freely across Wireless Local Area Network and the cellular network. The estimation of received signal strength may not always indicate the better quality of service. Hence packet loss rate is estimated to determine the occurrence of delay, jitter and contention problems and provide efficient and continuous transmission. The selection of best network having lowest packet loss rate during transmission helps us to improve the performance and also reduces network traffic and provides load balancing capability. The Vertical handoff algorithm is used to measure the packet loss and provides analytical framework for measuring the system performance. And hence optical relay assignment algorithm used to improve the network efficiency. The obtained results shows that loss, delay can be minimized and throughput can be increased.
\end{abstract}

Keywords: Vertical Handover(VH), heterogeneous network, mobile terminal(MT), WLAN/Cellular network.

\section{Introduction}

The development of the wireless and mobile technologies capable to rise the widespread coverage area, continuous end to end data service objectives, cost and acceptable level of quality of service[1],[3]. Handover is the mechanism by an ongoing connection between a Mobile terminal (MT) and a correspondent terminal(CT).

Handheld mobile terminal gradually integrate to operate WLAN and Cellular Network $(\mathrm{CN})$.Handover mechanism involves by allowing the ongoing voice call to continuously switching between the two interfaces. Thus the optimal results of vertical handover algorithm required [2], [4], [5]

This paper proposed the novel framework for calculating the packet loss ratio based on the VH algorithm by voice over internet protocol VOIP for the mobile users roaming between WLAN and Cellular Networks. The functionalities of the transport and the network are enabled by the handover execution mechanism (e.g. [6], [7]). This function determines the connection establishment and the handover triggering. The handover mechanism governs the handover process instantly required by ensuring the voice call seamless connectivity and maximizing the appropriated resource allocated by the available media (Fig.1.). However more handover algorithm exhibit in the literature, e.g. [8][12], these techniques mostly rely on poor performance evaluation. And basically depend on the signal strength determination. The methodology of such algorithm based on Received Signal Strength Indicator(RSSI) by the author analyzed previous work [13], [14].in [15] basically a PLR implemented and produce the initial results of simulation. In this paper, we addressed a thorough analysis of PLR-based performance characteristics of $\mathrm{VH}$ algorithm.

To trigger the handover execution a generic handover algorithm depends on the observed RSSI samples. When MT connects with WLAN it initiates handover from WLAN to the cellular network. For forwarding the voice call traffic from the service provider it takes a time. This technique is analyzed by the previous work [10], [13], [14], [16]. In reality received signal strength may not ensure adequate voice quality. Compare to Received Signal Strength Indicator (RSSI) the PLR can cause higher network impairments. The real time effects occur during the data transmission of voice call such as packet delay, jitter, and contention the PLR-based algorithm produces better performance and link assessment under these circumstances. The most of the existing works demonstrate e.g. [8]-[12], not prior given to the inter-networking environment of WLAN and $\mathrm{CN}$. In [15] proposed the performance evaluation by achieving a high WLAN usage rate with the parallel development of low outage rate in VH algorithm. In [17] the probability ratio of handover is modeled analytically by the mobile user roaming between the two integrated networks UMTS-WLAN. This paper does not analyze the requirements of $\mathrm{VH}$ algorithm or the outage probability in terms of the usage efficiency. In [18], to design a handover algorithm it considers a several QOS parameters and formulates the efficient network selection. The drawback of this paper is the signal strength is not analytically modeled.

The call drop occurs based upon the VoIP call over WLAN which depend on number of voice packet lost at the receiver side.

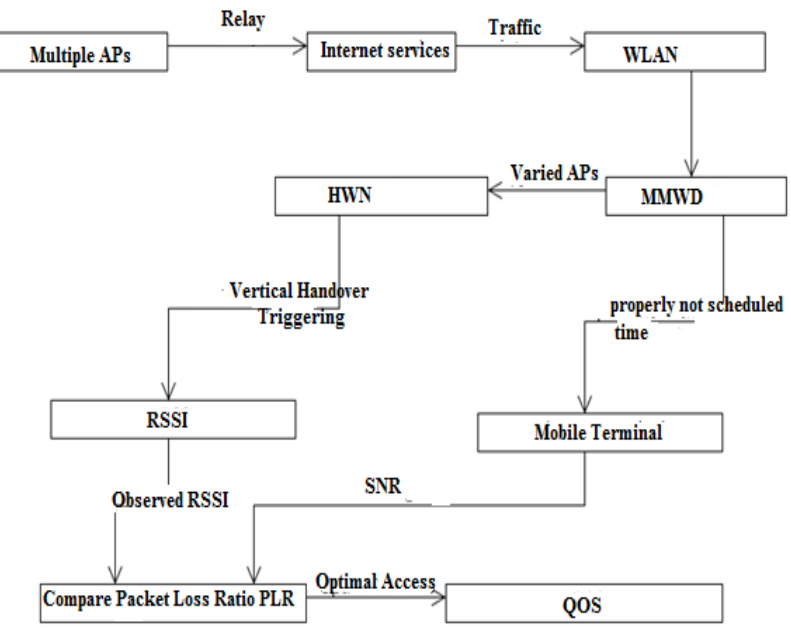

Figure 1: Back bone network

\section{Volume 5 Issue 6, June 2016 www.ijsr.net}




\section{International Journal of Science and Research (IJSR) \\ ISSN (Online): 2319-7064}

Index Copernicus Value (2013): 6.14 | Impact Factor (2015): 6.391

\section{System Analysis}

The multimodal wireless device (MMWD) allows user to depend on the heterogeneity function roaming across WLAN and Cellular Network that cover the area which experiences varying levels of signal strength from the different access points (AP) and base station(BS).the voice call over WLAN its quality depends upon the various parameters such as user mobility, signal strength attenuation, network congestion etc. these parameters causes packet losses and delays. The maximization of PLR which in turns reduces the voice quality level and also causes outage. The acceptable level of quality and seamless connectivity can performed effectively by triggering handover between the WLAN and cellular network by the $\mathrm{VH}$ algorithm. The functionalities of the $\mathrm{VH}$ algorithm and the heterogeneity aspects of wireless environment are presented below.

\section{A. Packet Loss Model In Voice Over Wlan/Cellular Network}

The fading effects such as small scale fading and large scale path loss which degrades the amount of signal strength attenuation over wireless transmission medium. In [19] the most used model for large scale path loss is the received power(in $\mathrm{dBm})$ at an MT with d meters away from the AP is,

$$
\mathrm{P}_{\mathrm{r}}(d)=P(t)-L-10 n \log \frac{d}{\mathrm{~d}^{*}}+\chi(\mu, \sigma)
$$

Where $\mathrm{P}_{\mathrm{r}}(d)$ Received power due to shadow fading in local mean, Lis a constant path loss (in $\mathrm{dB}$ ) at a distance $\mathrm{d}$ (meters) from the AP, $\mathrm{P}(\mathrm{t})$ is the transmitted power (in $\mathrm{dBm})$ and $\mathrm{n}$ is a path loss exponent. $\chi(\mu, \sigma)$ is a Gaussian random variable according for the shadowing (in $\mathrm{dB}$ ) with mean $\mu$.This results mobility of an object in the MT. this degrades the signal strength from local mean signal power. This drawback is overcome in [19], [20] by Rayleigh fading over the signal power.

The local mean strength of each location MT estimates the packet error probability. Hence by varying the time the MT exhibits the Packet Loss Pattern (PLP). Some of the packet experiences the small scale fading, and also some of the location determines the packet tends to lost randomly.

The probability of packet loss pattern (PLP) depends on the state of the previous packet. While the packet transmitted the received packet experiences by the two parameters $R$ and $S$, where $\mathrm{R}$ is denoted as the probability of next packet loss and $\mathrm{S}$ is denoted as the packet lost in the PPL, (Fig. 2). In general $\mathrm{S}$ will be higher than $\mathrm{R}$ this experience the burst errors. The average taken for the packet pattern loss is expressed as

$\mathrm{P}_{\text {loss }}=\frac{R}{R+1-S}$

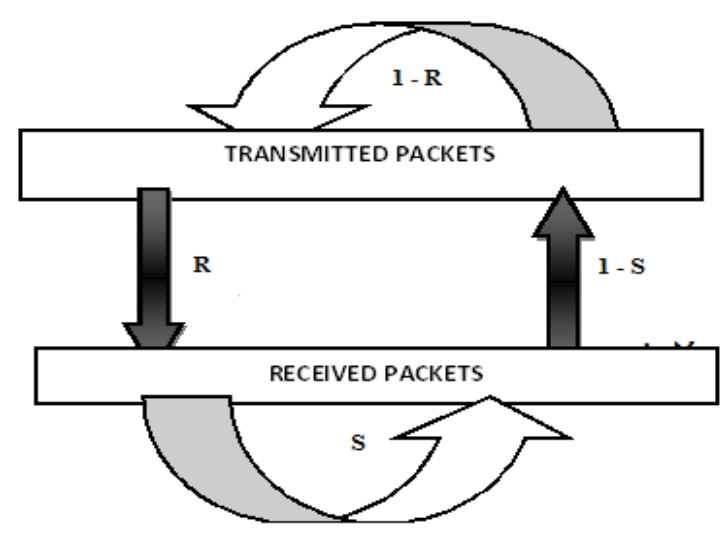

Figure 2: Packet Pattern Loss

The reason of user's mobility degrades the Fluctuation of the received signal power, this results the variation from the distance MT to AP. in general each location of the MT, and signal strength achieved PLR which depend on the physical layer characteristics. In [22] the voice quality is expressed in terms of average function of PLR. The PLR is directly proportional to the bit error rate(BER) of a transmission channel the reason of users mobility degrades the Fluctuation of the received signal power, this results the variation from the distance MT to AP. in general each location of the MT, signal strength achieved PLR which depend on the physical layer characteristics. In [22] the voice quality is expressed in terms of average function of PLR. In [23] the PLR is directly proportional to the bit error rate(BER) of a transmission channel. In [24] the monotonic function of signal-to-noise ratio(SNR) or the received signal strength is monotonically dependent on the bit error rate (BER) in the Rayleigh fading channel. The signal strength in terms determines the threshold level of call quality and call drop. In [13], [14] the threshold signal strength is specified below the call cannot be served over the WLAN interface. The call drop occurs while the signal strength falls below the level when the mobile terminal is connected to the WLAN.

This paper we demonstrate the call drop model is the direct derivative function of packet loss pattern. This tends to achieve better signal strength than the voice quality.by varying the local mean strength with the MT is moving across the regions which accounts by varying the PPL. When the MT degrades the coverage area of WLAN simultaneously the PPL will be increase. This cause the call drop or outage, when the handover is sufficiently not fast. Thus the call drops in terms of instantly with the packet loss ratio (PLR). By determining the period of time (T) with the packet loss rate $(\mathrm{L})$, this experience the threshold loss ratio of call drop(L1).

To determine the packet loss pattern we modeled call drop in rely with packet losses. The call drop monotonically dependson the various circumstances with varying VoIP execution. If a call drop occurs thiscriterion slowly degrades the quality, i.e. these implementations have less tolerance with the packet loss. At the same time the period of silence for few seconds some of the execution which does not exceeds the call drop. This accounts the average amount of packet loss ratio determines the seamless continuity and efficient quality of a VoIP call, this shows the enormous difference in the quality of the predetermine call quality 


\section{International Journal of Science and Research (IJSR) \\ ISSN (Online): 2319-7064 \\ Index Copernicus Value (2013): 6.14 | Impact Factor (2015): 6.391}

simultaneously at the same PLR, for different loss specified. The criteria of voice packets have inter-dependence among the loss patterns, either the loss of one or two packets in a block wise packet loss. This accounts by not affecting the voice quality. In [25] modeled the various quality states with the Markov models. In [15] the call drop depends upon the number of packets lost over a specific period of time. Finally, this accounts by experimentally the packet loss patterns causing an outage.

For the usage of reconstruction speech signal the delayed voice packets are received after a certain threshold level this specified the voice packets are lost. Before specified the packets arrive and received experiences the probability less than 1 . The resultant packet loss probabilities $\mathrm{R}$ and $\mathrm{S}$ are considered throughout this paper to determine the voice packet from the voice codec perspective. This paper deals with the characterization of the several probabilities in terms of network dynamics, wireless environment, user mobility are beyond the scope. This perceived the future enhancement.

\section{B. Handover Triggering Algorithm}

In [26] [27] to decide whether and when to initiate the handover a generic handover algorithm required this relies on the received signal and indicate by the RSSI received signal strength indicator. To perceived the condition of the WLAN interface the different patterns of the RSSI samples used. The triggering of handover from WLAN to cellular network operation is predefined by the RSSI samples by the condition when MT is connected to the WLAN. The handover triggering algorithm trigger the handover process by taking a specific amount of time (t1) to execute the handover. This concealed the forwarding voice traffic by using the new interface. For example in [16] the algorithm triggers the handover by certain amount of perceived RSSI samples this falls by the given threshold. The mobile nodes or the mobile terminal receives the various signal strength from different wireless heterogeneous wireless networks.

In general the strong received signal strength may not necessarily achieve adequate voice quality. The network impairments computes and compare to RSSI suggest higher amount of PLR. In existing RSSI algorithm is an important class of algorithm but the proposed PLR based algorithm analyzed to achieve better performance under most distortion [15]. The better link assessment can appropriately achieve by PLR than RSSI by computing the real time effects of jitter, packet delay and contention. Thus in reality we evaluating packet lost based algorithm in this paper.

The packet lost based algorithm the handover experience the packet loss percentage (12) and specific period of time(t2). If the packet loss (12) exceeds (L1) of handover threshold loss, then the call is handover to the cellular network in accordance with the (11) this soon exceeds the handover threshold loss (L1). The probability of the call drop rate is calculated when MT implemented under this algorithm. The main aim of a $\mathrm{VH}$ algorithm is to make an optimal result of WLAN interface whenever it is sufficient. In [13], [14], [15] proposed by evaluating the performance metric by the handover algorithm parameters and WLAN usage efficiency. The WLAN usage efficiency can be calculated as the ratio of total amount of period required of MT to be connected with the WLAN interface (TWLAN-used) to the ratio of the MT capable to usable the amount of (WLANusable)

$\eta$ WLAN $=\frac{\text { TWLAN }- \text { used }}{\text { TWLAN }- \text { usable }}$

The WLAN tends to operate with the amount of bandwidth available with its good signal strength for connectivity. The main objective is to compute to produce better call efficient quality at a cheaper price. By the preferred network choice the WLAN usable and used determined. However maximize the amount of $\eta$ WLAN enables to achieve better utilization of the WLAN this is implemented while designing the $\mathrm{VH}$ algorithm. Simultaneously the desirable call drop probability needs pre specified threshold at the same time. The VH algorithm's depend upon the two main parameters they are $\eta \mathrm{WLAN}$ and probability of call drop. The main objective is to perform by higher value of $\eta W L A N$ and by minimizing the probability of call drop to perform the operation of $\mathrm{VH}$ algorithm more effectively.

\section{Performance Evaluation}

\section{A. The Fundamental Handover Of Cellular Networks}

Handover process is transferring an active call from one cell to another. The transfer of current communication channel could be in terms of time slot, frequency band, or code word to a new base station (BS), which leads to different techniques of handover. If new BS has some unoccupied channels then it assigns one of them to the handover. If all of the channels are in use at the handover time there are two possibilities: to drop the call or delay it for a while. In order to evaluate the efficiency of handover, two of the most important metrics for evaluating a handover technique are forced termination (call dropping) probability and call blocking probability. The forced termination probability is the probability of dropping an active call due to handover failure and the call blocking probability is probability of blocking a new call request.

The aim of a handover procedure is to decrease forced termination probability while not increasing call blocking probability significantly in (Fig.3)

The mobile nodes into a different cell while conversation is in process Auctioneer or backbone network automatically transfer the call to new channel belonging to new BS $[1,2]$ The advent of handover involves identifying a new BS, but also requires the voice and control signal be allocated to channel associated with new BS. The parallel development of the network provided the mobile users with the enormous amount of continuous sufficient data and voice communication in different network and of varying time.

The instant neatly time $\left(\mathrm{t}_{\text {start }}\right)$ implies the call active in the case of WLAN properly connected with the MT. This exploitation indicates the call handover to the WLAN at the time interval of $\left(t_{\text {start }}\right)$, or either the at the first place of the time interval $\left(t_{\text {start }}\right)$ the call been initiated by the WLAN.The successful exploitation of first call drop instantly occur is represent as $\left(t_{d r o p}\right)$ or either the handover at the out of coverage instantly is represent as $\left(t_{\text {hand-out }}\right)$ from the instant time at $\left(\mathrm{t}_{\text {start }}\right)$.

\section{Volume 5 Issue 6, June 2016}

www.ijsr.net 


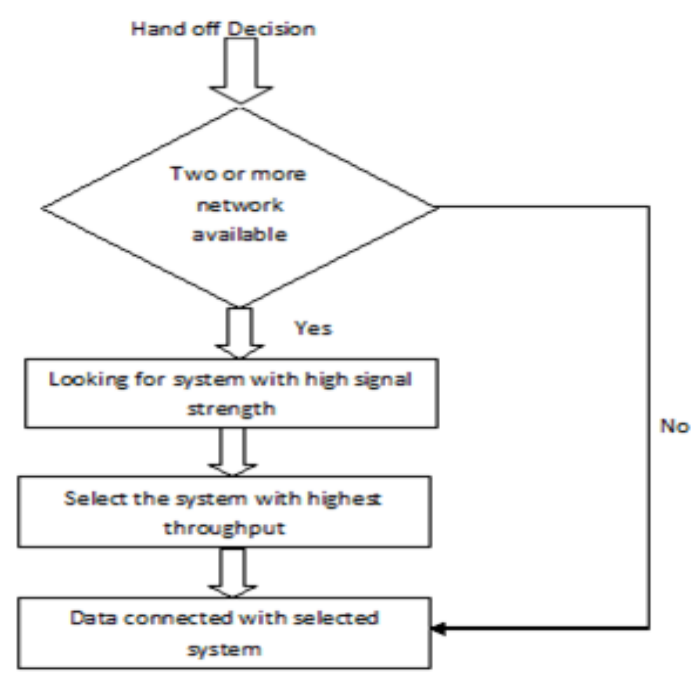

Figure 3: Handover decision

Moreover the out of coverage handover at a time ( $\left.\mathrm{t}_{\text {hand-out }}\right)$ occur while the handover time $\left(t_{\mathrm{HO}}\right)$ or either $\left(\mathrm{t}_{\mathrm{drop}}\right)$ the objective of this case is successful transmission of call been forwarded safely without any call drop. Thus at the starting time of the call drop probability is represented as,

$\operatorname{Prob}\left(\mathrm{C}_{\mathrm{dr}} \mid \mathrm{t}_{\mathrm{star}}\right)=1-\operatorname{Prob}\left(\mathrm{t}_{\text {hand-out }} \leq \mathrm{t}_{\mathrm{drop}}-\mathrm{t}_{\mathrm{HO}} \mid \mathrm{t}_{\text {start }}\right)$

The $\left(\mathrm{C}_{\mathrm{dr}}\right)$ implies the call drop event. This resembles $\left(t_{\text {drop }} \geq t_{\text {start }}\right)$ with the instant time $\left(t_{\text {drop }}\right)$ with either fixed or vary with the relevant distributions.

\section{B. Horizontal handover algorithm for homogeneous wireless networks}

The horizontal handover decision presented in this paper is based on genetic algorithm from the service provider it receives the no traffic flow of information this communicate with the WLAN through BPLP block packet loss pattern protocol is given to horizontal handover by using same AP in (Fig.4). The received signal is observed by the indicator RSSI received signal strength indicator

The horizontal comprises of 3 links upper link, down link and drop link. In upper link if one packet send on the particular area, minimum traffic exhibit so the delay ratio reduced in upper link. In down link the traffic affects and also the data tends to loss more as a result the maximum delay occurs. In drop link it checks the mobile terminal (MT) to stay connected with the received signal strength indicate by the indicator known as received signal strength indicator (RSSI) and also used to calculate the packet loss ratio (PLR). The MAC protocol comprises of CSMA, RTS, CTS and the data link layer for the required data to be transmit effectively. In horizontal handover if the out of coverage exhibit when the service provider to reach destination this results simple packet loss so drop occurs. TCP used for connect oriented protocol used to transfer the packets from source to destination. FTP is used for file transfer it is based on connect oriented protocol and UDP is used for connectionless, during sending the dat or file it remains as connect while of out of range it resembles connection less so the call drop occurs.
Thus in the horizontal handover algorithm for homogeneous wireless networks many drawbacks occurs such as lack of paging support i.e. user preference support [3], effects on long update latency so the vertical handover algorithm implemented by the algorithm prony.Prony algorithm can be implemented in both homogeneous and heterogeneous networks will be nonlinear function used to determine WLAN available, usable and used.

\section{Formation Of Heterogeneous Wireless Networks}

Wireless communications infrastructure over the last few decades has given rise to a multitude of wireless networks with varying degrees of coverage, service objectives, pricing and quality of service. While the parallel development of these networks has provided the users with essential data and voice communication options at different levels at different times, it has also yielded a somewhat redundant heterogeneous mix of coexistent dissimilar networks that often suffers from bandwidth scarcity and poses additional monetary burdens on the end users.

Consider it as ubiquitous, while the WLAN offers its services over a rather limited region. The WLAN has one AP and if the MT is disassociated from it, there is no other AP to connect to. The signal strength attenuation over a wireless transmission medium is caused both by the large scale path loss and the small scale fading and the MT is using the WLAN interface for packet transmission, the effects of the impairments caused by small-scale fading can be sufficiently At each location the MT occupies along its trajectory, the local mean signal strength determines the packet error probability at that instant. Therefore, the MT experiences a packet loss pattern that varies over time. The uncertainty of a packet being received stems from the smallscale fading. Even at a location with a strong local mean signal power, packets can be lost randomly.

\section{Vertical Handover Algorithm for Heterogeneous Wireless Networks}

A generic handover algorithm relies on the observed RSSI and when to initiate a handover The RSSI samples is used in assessing the condition of the WLAN interface. When an MT is connected to the WLAN, it triggers a handover from WLAN to cellular. The predefined RSSI derivative meets a certain condition. Once the handover triggering algorithm triggers a handover, it takes a certain amount of time to complete the handover, and start forwarding voice traffic using the new interface. The algorithm triggers a handout if a certain number of consecutive RSSI samples fall below a given threshold. In heterogeneous wireless networks (Fig 4), a mobile node receives various signal strengths from different networks. The algorithms in computes the moving average of the RSSI values and compares.

The horizontal handover decision presented in this paper is based on genetic algorithm from the service provider it receives the no traffic flow of information this communicate with the WLAN through BPLP block packet loss pattern protocol is given to horizontal handover by using same AP in (Fig.4). The received signal is observed by the indicator RSSI received signal strength indicator 


\section{International Journal of Science and Research (IJSR) \\ ISSN (Online): 2319-7064}

Index Copernicus Value (2013): 6.14 | Impact Factor (2015): 6.391

To overcome the effects of long update latency, high signaling overhead, lack of paging support i.e. user preference support [3] the Vertical handover algorithm implemented by the algorithm prony.Prony algorithm can be implemented in both homogeneous and heterogeneous networks will be nonlinear function used to determine WLAN available, usable and used.

This average is determined as threshold. This is the class of algorithms we analyzed under our performance analysis framework in. However, strong received signal strength, in reality, may not necessarily ensure an adequate voice quality. Network impairments can cause a higher PLR than the RSSI suggests. Although RSSI driven algorithms still are an important class of algorithms, PLR-based algorithms can demonstrate better performance under most circumstances. Real-time effects of packet-delay, jitter, and contention can potentially be helpful in making a better link assessment, which the RSSI alone cannot provide

The MT experiences the fading effects and the mobility parameters in the system model with the time $\left(t_{\text {drop }}\right)$ and $\left(t_{\text {start }}\right)$ with the variation signal strength and packet loss pattern. Thus the vertical handover in the heterogeneous wireless networks probability can be expressed as,

${ }^{\operatorname{Prob}\left({ }^{t}\right.}$ hand-out $\leq\left.{ }^{\mathrm{t}} \mathrm{drop}^{-{ }^{\mathrm{t}} \mathrm{HO}}\right|^{\mathrm{t}}$ start ${ }^{\text {' }}$

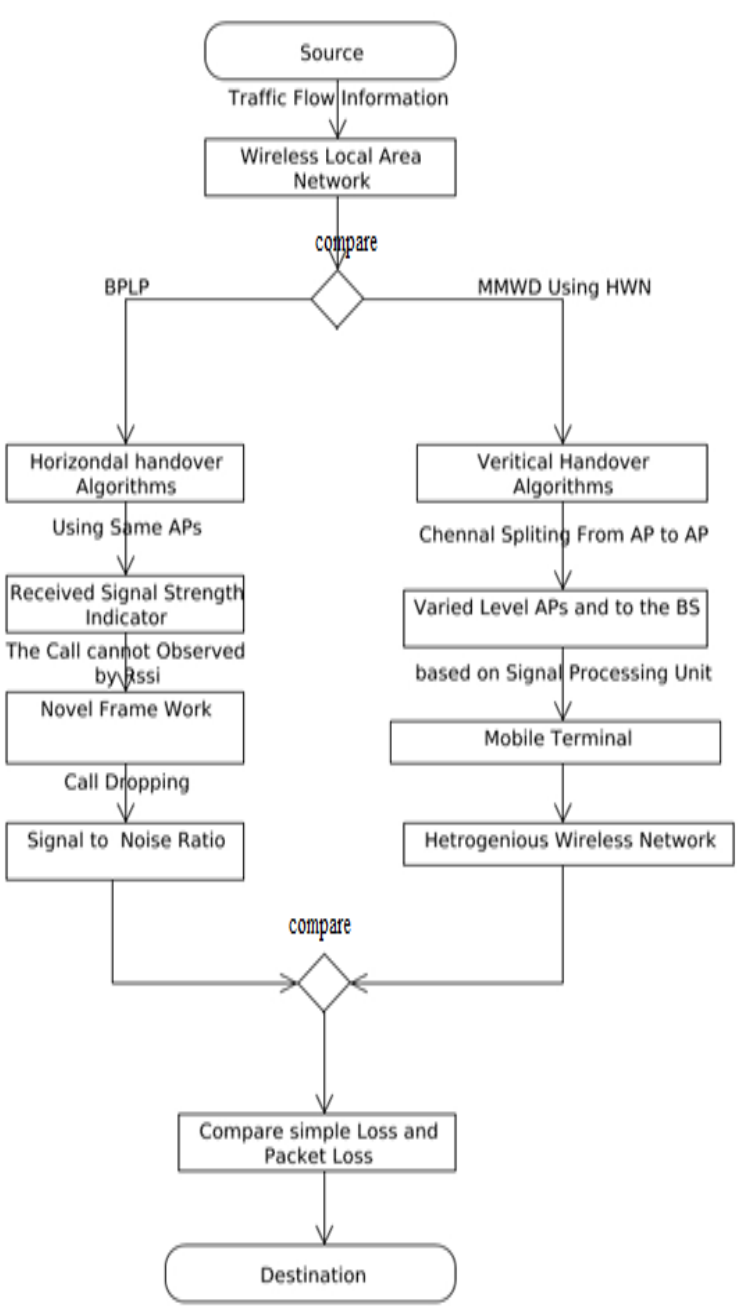

Figure 4: Flow diagram

\section{Optimal Cooperative Communication}

The cooperative communication is equipped with the single transceiver the objective is to exploit with the another cooperative mobile node in the network to perform cooperative communication effectively. This performs the linear operation on the signal strength received from the service provider obtained source of information is been forwarding to the destination node. Before forwarding the call the cooperative node decode and encode the received signal then fetch to selecting the sequence with the required destination. In the network environment the relay assignment problem occurs. In that case the multiple active source-destination pairs and all other nodes act as relay node or intermediate node. This is able to offer power full communication with the reduce losses. The performance is of this communication is based on the selection of the relay node, thus the number of relay node in the network is guaranteed for each source-destination pair to have capacity no less than direct transmission and its ability to find the optimal objective regardless of initial assignment. The benefit of cooperative communication possible for a sourcedestination pair to employ multiple relay nodes this also provides the best relay to achieve full efficient transmission to have multiple relay node participate. Optimization of relay node selection is the main objective for cooperative communication and resource allocation in the cellular network. Optimal Relay Assignment includes

a) Whether the relay nodes in the network is more than or less than the number of source-destination pairs;

b) The final capacity for each source-destination pair is guaranteed to be no less than that under direct transmissions;

c) The algorithm is able to find the optimal objective regardless of initial relay node assignment.

\section{Output Screenshots and Simulation Result}

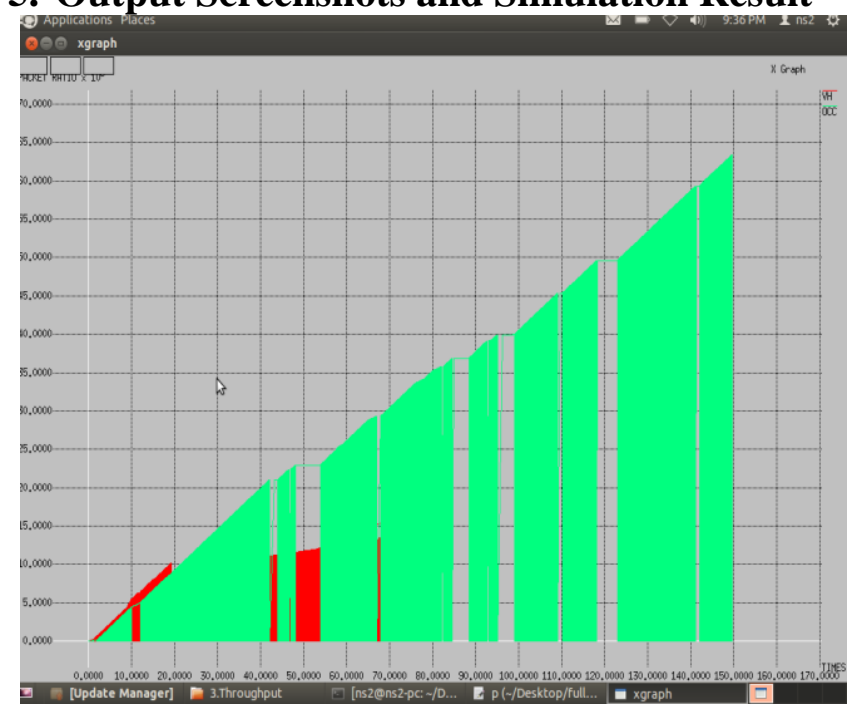

Figure 5: Output graph for Throughput between existing system and proposed system 


\section{International Journal of Science and Research (IJSR) \\ ISSN (Online): 2319-7064}

Index Copernicus Value (2013): 6.14 | Impact Factor (2015): 6.391

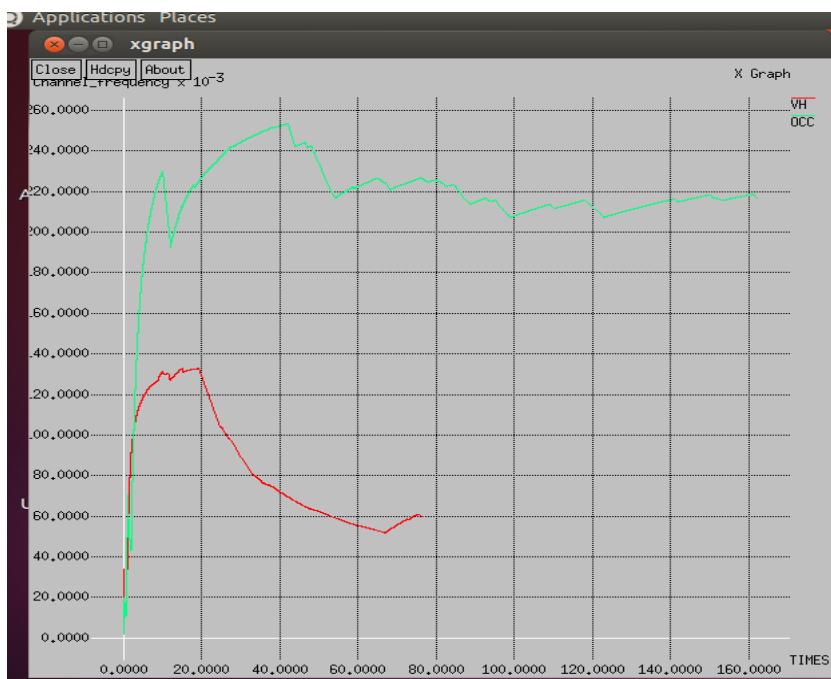

Figure 6: Output graph for channel Measurement between existing system and proposed system

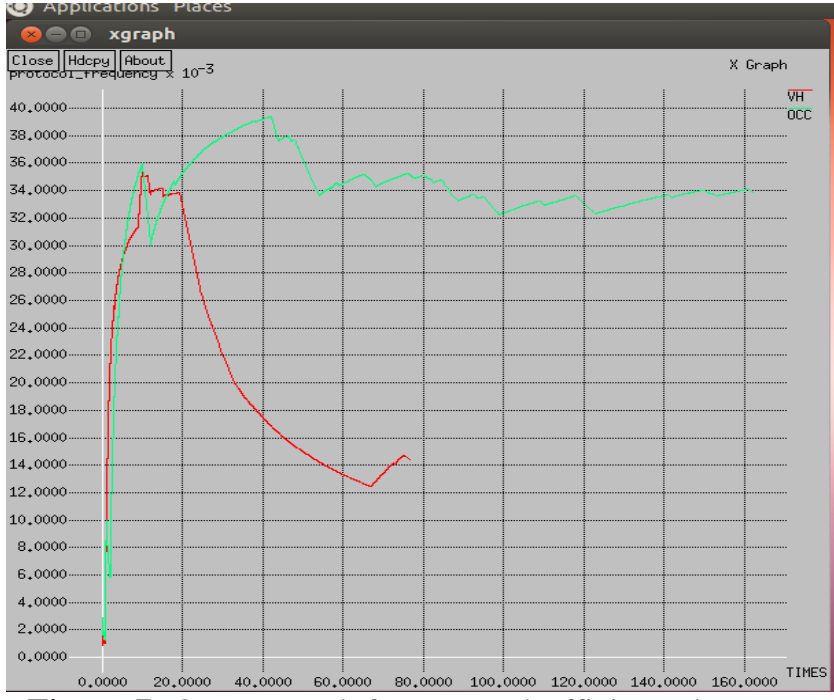

Figure 7: Output graph for Protocol Efficiency between existing system and proposed system

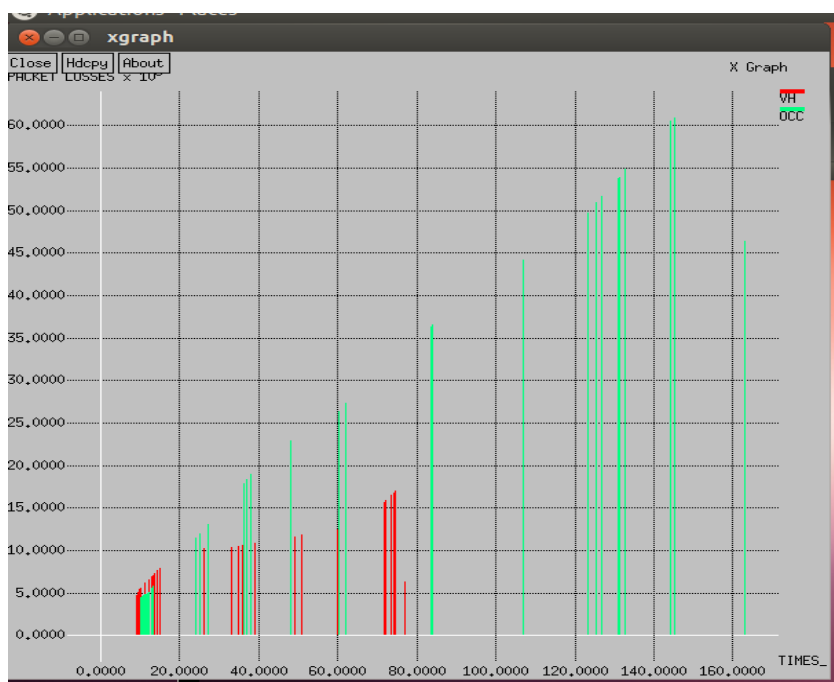

Figure 8: Output graph for Packet Loss Ratio between existing system and proposed system

\section{Conclusion}

This paper proposes the performance evaluation of packet loss based VH algorithm for voice over WLAN and cellular network. The packet loss rate of $\mathrm{VH}$ algorithm is based upon the amount of moving average of the packet losses. The call drop or outage is monotonically depend on the function of packet loss rate. Based upon the varying system and handover threshold shown with the performance trends of WLAN usage efficiency and call drop probabilities. This provides handheld mobile terminal in heterogeneous network maintains a seamless connectivity and acceptable level of quality. The analysis of packet loss based algorithm for an internetworking environment which compromise the WLAN and cellular network. Thus PLR serves a more reliable indicators and more efficient with the different combination of networks. By the cooperative communication plays important role to improve the overall system performance. Thus by finding optimal results by assigning relay nodes to the source and destination pairs so as to maximize the capacity among all pairs of different combination in the nearby networks. Thus the cooperative communication and resource allocation appropriately well defined in the cellular network. Thus cooperative communication is a powerful communication. however the performance of such communication used to select the relay node in a network environment., where multiple source destination pairs compete for the same pool of relay nodes in the network, its guarantee for each source and destination pair to have capacity no less than direct transmission and its ability to find the optimal objective regardless of initial assignment.

\section{References}

[1] Tariq Ali, "Analysis of an Instantaneous Packet Loss Based Vertical Handover Algorithm for Heterogeneous Wireless Networks," IEEE Trans. Mobile Computing., vol. 13, no. 5, pp. 992-1006., May 2014.

[2] Priti B Ghormade, Jagruti J.Shah, “Approach to perform horizontal and vertical handoff in wireless," IEEE Conf. ICACEA., pp.812-815., March 2015.

[3] D. Niyato and E. Hossain, "Dynamics of network selection in het-erogeneous wireless networks: An evolutionary game approach," IEEE Trans. Veh. Technol., vol. 58, no. 4, pp. 2008-2017, May 2009.

[4] H. Chan, P. Fan, and Z. Cao, "A utility-based network selection scheme for multiple services in heterogeneous networks," in Proc.IEEE Int. Conf. IWCMC, vol. 2. Jun. 2005, pp. 1175-1180.

[5] M. Lott, M. Siebert, S. Bonjour, D. von Hugo, and M. Weckerle, "Interworking of WLAN and 3G systems," IEE Proc. Commun., vol. 151, no. 5, pp. 507-513, Oct. 2004.

[6] R. Chakravorty, P. Vidales, K. Subramanian, I. Pratt, and J. Crowcroft, "Performance issues with vertical handovers - Experiences from GPRS cellular and WLAN hot-spots integra-tion," in Proc. 2nd IEEE Annu. Conf. Pervasive Computing andCommunication, Mar. 2004, pp. 155-164.

[5] F. Zhu and J. McNair, "Optimizations for vertical handoff decision algorithms," in Proc. IEEE WCNC,

\section{Volume 5 Issue 6, June 2016} www.ijsr.net 


\section{International Journal of Science and Research (IJSR) \\ ISSN (Online): 2319-7064}

Index Copernicus Value (2013): 6.14 | Impact Factor (2015): 6.391

2004.

[6] H. J. Wang, R. H. Katz, and J. Giese, "Policy-enabled hand-offs across heterogeneous wireless networks," in Proc. MobileComputing Systems and Applications, New Orleans, LA, USA, 1999.

[7] A. H. Zahran and B. Liang, "Performance evaluation framework for vertical handoff algorithms in heterogeneous networks," in Proc. IEEE ICC, 2005.

[8] M. Ylianttila, M. Pande, J. Makela, and P. Mahonen, "Optimization scheme for mobile users performing vertical handoffs between IEEE 802.11 and GPRS/EDGE networks," in Proc. IEEE Global Telecommunications Conf., San Antonio, TX, USA, Nov. 2001.

[9] S. Xie and M. Wu, "Vertical handoff algorithm in heterogeneous networks for reducing interference," $J$. Electron., vol. 26, no. 1, pp. 71-79, Jan. 2009.

[10]T. Ali and M. Saquib, "Analytical framework for WLAN-cellular voice handover evaluation," IEEE Trans. Mobile Comput., vol. 12, no. 3, pp. 447-460, Mar. 2013.

[11]T. Ali and M. Saquib, "Performance evaluation of WLAN/cellular media access for mobile voice users under random mobility mod-els," IEEE Trans. Wireless Commun., vol. 10, no. 10, pp. 3241-3255, Oct. 2011.

[12] T. Ali, M. Saquib, and C. Sengupta, "Performance analysis frame-work and vertical handover triggering algorithms for voice over WLAN/cellular network," in Proc. IEEE Wireless Communicationand Networking Conf., Las Vegas, NV, USA, Apr. 2008.

[13] C. W. Lee, L. M. Chen, M. C. Chen, and S. S. Yeali, “A framework of handoffs in wireless overlay networks based on mobile IPv6," IEEE J. Select. Areas Commun., vol. 23, no. 11, pp. 2118-2128, Nov.2005.

[14] H. Bing, C. He, and L. Jiang, "Performance analysis of vertical handover in a UMTS-WLAN integrated network," in Proc. IEEEInt. Symp. Personal, Indoor and Mobile Radio Communication, Sep.2003.

[15] E. Stevens-Navarro, Y. Lin, and V. W. S. Wong, “An MDP based vertical handoff decision algorithm for heterogeneous wireless networks," IEEE Trans. Veh. Technol., vol. 57, no. 2, pp. 1243-1254, Mar. 2008

[16] T. S. Rappaport, Wireless Communications: Principles and Practice. Upper Saddle River, NJ, USA: Prentice Hall, 2002.

[17]D. Wong and D. Cox, "Estimating local mean signal power level in a Rayleigh fading environment," IEEE Trans. Veh. Technol., vol. 48, no. 3, pp. 956-959, May 1999.

[18] J. Zhang and D. Yang, "Quality of VoIP in wireless commu-nication networks," in Proc. IEEE Wireless Communications andNetworking Conf., Las Vegas, NV, USA, 2006

[19] O. Awoniyi and F. Tobagi, "Effect of fading on the perfor-mance of VoIP in IEEE 802.11 WLANs," in Proc. IEEE Int. Conf.Communications, 2004.

[20] P. Fuxjager and F. Ricciato, "Collecting broken frames: Error statis-tics in IEEE $802.11 \mathrm{~b} / \mathrm{g}$ links," in Proc. 6th Int. Symp. Modelingand Optimization in Mobile, Ad Hoc, and Wireless Networks and Workshops, Berlin, Germany, pp. 30-35, Apr. 2008.

[21]J. G. Proakis, Digital Communications. Boston, MA, USA: McGraw-Hill, 2001.
[22] T. Braskich, N. Smavatkul, S. Emeott, and T. Wilsont, "Link adap-tation evaluation for WLAN using a voice quality metric," in Proc.IEEE Global Telecommunications Conf., Dec. 2004.

[23] G. P. Pollini, "Trends in handover design," IEEE Commun. Mag., vol. 34, no. 3, pp. 82-90, Mar. 1996.

[24] P. Marichamy, S. Chakrabarti, and S. L. Maskara, "Performance evaluation of handoff detection schemes," in Proc. IEEE Conf.Convergent Technologies for the Asia-Pacific Region, Oct. 2003.

[25] K. Pahlavanet al., "Handoff in hybrid mobile data networks," IEEE Pers. Commun., vol. 7, no. 2, pp. 3447, Apr. 2000.

[26] R. Ben Ali and S. Pierre, "Optimal voice admission control perfor-mance under soft vertical handoff in loosely coupled 3G/WLAN networks," in Proc. IEEE Wireless Communications and NetworkingConf., 2008.

[27] L. Hua, M. H. Kabir, and T. Sato, "Velocity adaptive vertical hand-off on multi-frequency system," in Proc. IEEE Int. Symp. Personal,Indoor and Mobile Radio Communications, Tokyo, Japan, 2009. 